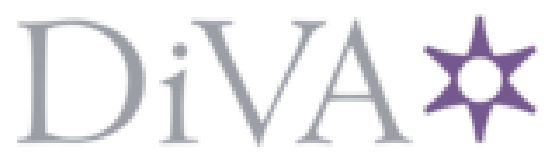

http://www.diva-portal.org

\title{
Postprint
}

This is the accepted version of a paper published in Wind Energy. This paper has been peer-reviewed but does not include the final publisher proof-corrections or journal pagination.

Citation for the original published paper (version of record):

Ottermo, F., Bernhoff, H. (2014)

An upper size of vertical axis wind turbines.

Wind Energy, 17(10): 1623-1629

http://dx.doi.org/10.1002/we.1655

Access to the published version may require subscription.

N.B. When citing this work, cite the original published paper.

Permanent link to this version:

http://urn.kb.se/resolve?urn=urn:nbn:se:hh:diva-25140 


\title{
Short Communication
}

\section{An upper size of vertical axis wind turbines}

\author{
F. Ottermo ${ }^{1,2}$ and H. Bernhoff ${ }^{1,3}$ \\ ${ }^{1}$ Division for Electricity, Department of Engineering Sciences, Uppsala University, Box 534, SE-751 21 Uppsala, Sweden \\ ${ }^{2}$ School of Business and Engineering, Halmstad University \\ ${ }^{3}$ Swedish Centre for Renewable Electric Energy Conversion
}

\section{ABSTRACT}

The scaling behaviour of a straight-bladed vertical axis wind turbine is considered. A scaling scheme is described that, in the presence of a wind shear profile, aims at leaving the material stresses of the scaled construction unchanged. Based on a recent $200 \mathrm{~kW}$ three-bladed H-rotor design, a structural upper size of the turbine is proposed, this size being the scale at which the gravitational force starts to become important. As gravity has a much worse scaling behaviour than the aerodynamic and centrifugal forces, the construction work will become increasingly more difficult above this scale. The upper size is estimated to be around 30 MW. Copyright (c) 0000 John Wiley \& Sons, Ltd.

\section{KEYWORDS}

VAWT, H-rotor, scaling, upper size

Correspondence

F. Ottermo, Div for Electricity, Dept of Engineering Sciences, Uppsala University, Box 534, SE-751 21 Uppsala, Sweden. E-mail: fredric.ottermo@hh.se

Received...

\section{INTRODUCTION}

Competitiveness is likely to be a prerequisite for wind power to become a substantial contributor to the global energy production and a relevant player to cut greenhouse gas emissions. In this effort the present direction is towards larger machines, which has been shown to make energy production both cheaper and greener [1]. However, for conventional horizontal axis wind turbines further reduction in cost of energy due to up-scaling relies on continuous technology breaktroughs concerning blade manufacturing and materials [2], break-throughs that are not a priori certain to take place. One example that is likely to push the size limit upwards is the concept of smart rotor control [3].

A promising and totally different approach to reduce the lifetime cost of wind power may be found in the concept of vertical axis wind turbines (VAWTs), where the number of moving parts can be kept at a minimum and the generator can be placed at ground level $[4,5]$. In this note we consider a specific VAWT design - the so-called H-rotor which has straight blades, connected by struts to a central hub on a tower. It is believed that up-scaling will be beneficial for this concept as well, and it is therefore interesting to study if there is a scale above which the gravitational loads will become dominant and structural design increasingly challenging. Such a turning point will constitute a structural upper scale of this type of VAWT. 
VAWTs appear to be better suited for large scales than conventional horizontal axis machines, as noted in, for example $[6,5]$. For horizontal axis wind turbines, gravity will impose a cyclic stress which, due to fatigue issues, will multiply its impact on material dimensioning. For VAWTs the only cyclically varying force is the aerodynamic force, which has a better scaling behaviour. This is likely to place VAWTs in a better position for up-scaling.

A typical design of an H-rotor, a three-bladed $200 \mathrm{~kW}$ turbine, is presented in [7]. This turbine was constructed with a focus on up-scalability and will constitute a reference design for the calculations presented here.

\section{SCALING MODEL}

This note considers scaling implications of the H-rotor, based solely on an analysis of loads on the strut joints. This means that many important aspects are omitted. Loads on the hub, bearings and tower are disregarded, as well as dynamic stall and control issues. Also omitted are transportation considerations, which, at very large scales, may have strong implications on the design.

The current analysis is based on the assumption that the turbine is geometrically similar when scaled, the material is unchanged and the nominal tip speed ratio is held constant. At first approximation, this type of scaling is reasonable as the dominant forces (the aerodynamic and centrifugal forces) scale as the area and leave the material stresses unchanged. However, in reality wind shear changes the scaling of the forces and we need to modify the scaling scheme to account for this, as presented below. The scale factor $\alpha$ is defined as the ratio of the scaled turbine radius $\tilde{R}$ to the nominal radius $R$,

$$
\alpha=\frac{\tilde{R}}{R}
$$

In what follows, the scaled quantity will be denoted by a tilde. All lengths of the turbine are assumed to scale as $\alpha$. For an H-rotor with radius $R$ and rotational frequency $\Omega$ in a wind field with unperturbed velocity $U$, the tip speed ratio is $\lambda=\Omega R / U$. Constant $\lambda$ at different scales means that the distribution of angles of attack during the revolution is close to identical; as a consequence, the aerodynamic forces per area will at first approximation be scale invariant (i.e. ignoring the change in Reynolds number). Assuming a power law profile for the wind speed with exponent $n=1 / 7$, the wind speed at the hub for the scaled turbine is $\tilde{U}=\alpha^{n} U$, as the hub height scales as $\alpha$. The rotational frequency then scales according to $\tilde{\Omega}=\alpha^{n-1} \Omega$. The power coefficient $C_{P}$ of a wind turbine is defined as

$$
P=\frac{1}{2} C_{P} \rho S U^{3}
$$

where $P$ is the generated power, $\rho$ is the density of air and $S$ is the cross-section area of the turbine. The maximum power coefficient $C_{P}$ at optimum $\lambda$ is assumed to be scale independent (even if we generally expect a slightly better value for larger turbines due to higher Reynolds number, since the increased turbulent content in the boundary layer delays flow separation [8]). Hence, according to (2), assuming constant $C_{P}$ the power absorption scales as,

$$
\tilde{P}=\alpha^{2+3 n} P \text {. }
$$

The aerodynamic forces $F_{a}$ on the blades are proportional to the blade area and the square of the wind speed, so that

$$
\tilde{F}_{a}=\alpha^{2+2 n} F_{a}
$$

As we will see, it is mainly the aerodynamic force that will govern the dimensioning for small scales. The material stress $\sigma$ in a joint (or piece of material) is proportional to $F / A$, where $F$ is the force on the joint and $A$ is the joint cross-section area. At strict similar scaling, the joint area scales as $\tilde{A}=\alpha^{2} A$. Since the aerodynamic force scales somewhat faster than $\alpha^{2}$ and we want to keep the material stresses constant, we assume that the wall thickness throughout the construction is 
Table I. Data for the $200 \mathrm{~kW}$ reference turbine

\begin{tabular}{ll}
\hline \hline Radius $(R)$ & $13 \mathrm{~m}$ \\
Hub height & $40 \mathrm{~m}$ \\
Blade count & 3 \\
Blade area (one blade) $\left(S_{b}\right)$ & $20 \mathrm{~m}^{2}$ \\
Blade mass (one blade) & $520 \mathrm{~kg}$ \\
Struts mass (for one blade) & $1 \times 10^{3} \mathrm{~kg}$ \\
Nominal wind speed $(U)$ & $12 \mathrm{~m} / \mathrm{s}$ \\
Nominal tip speed ratio $(\lambda)$ & 3.8 \\
Nominal RPM & 33 \\
Power coefficient $C_{P}$ & $\sim 0.35$ \\
Control method & Passive stall \\
\hline
\end{tabular}

scaled to maintain a constant relation between the total cross-section area and the aerodynamic force, i.e. $\tilde{A}=\alpha^{2+2 n} A$. The outer dimensions, however, are assumed to scale according to similarity. With unchanged material this implies that the rotor mass will scale as $\tilde{m}=\alpha^{3+2 n} m$. (Hence, as often mentioned in the context of wind turbines, the mass of the turbine grows faster than the power absorption when scaled up.) The centripetal acceleration $a$ experienced by the blades is $a=R \Omega^{2}$ and scales as $\tilde{a}=\alpha^{2 n-1} a$. The same holds for any position along the struts. As a consequence, the force $F_{c}=m a$ that is due to the centripetal acceleration scales as

$$
\tilde{F}_{c}=\alpha^{2+4 n} F_{c}
$$

Finally, all gravitational forces $F_{g}$ scales as $m$, so that

$$
\tilde{F}_{g}=\alpha^{3+2 n} F_{g}
$$

As expected, the gravitational force has the worst scaling behaviour of these forces. It is then clear that material stresses will, when scaled up, eventually become very large due to gravitational forces. However, for small turbines the centrifugal and aerodynamic forces will be dominant and determine the dimensions. At some point during scale up, the gravitational force becomes of comparable influence at key joints. The scale at which this happens may be considered as the structural upper scale of the turbine design under investigation. We will now look more closely at what is the upper scale of the Hrotor design. For nominal reference in the calculations, the $200 \mathrm{~kW}$ turbine described in [7] is used. This turbine is depicted in Figure 1 and data is given in Table I. However, as the turbine blades are constructed from fiberglass composites, and considering that at least $30 \%$ weight reductions may be expected by using carbon fiber instead [9], it is reasonable to reduce the blade and strut masses accordingly in this upper scale estimate. The blade weight that will be used in the calculations that follow is $350 \mathrm{~kg}$.

\section{MODEL IMPLICATIONS AND UPPER SCALE}

To illustrate the different forces that are considered here, Figure 2 schematically depicts a simplified strut design, where key joints have been numbered. A realistic design (as the one in Figure 1), usually includes two struts for each blade (or additional struts close to the blade), but for the concepts in this presentation it is sufficient to consider just one strut as in Figure 2. 


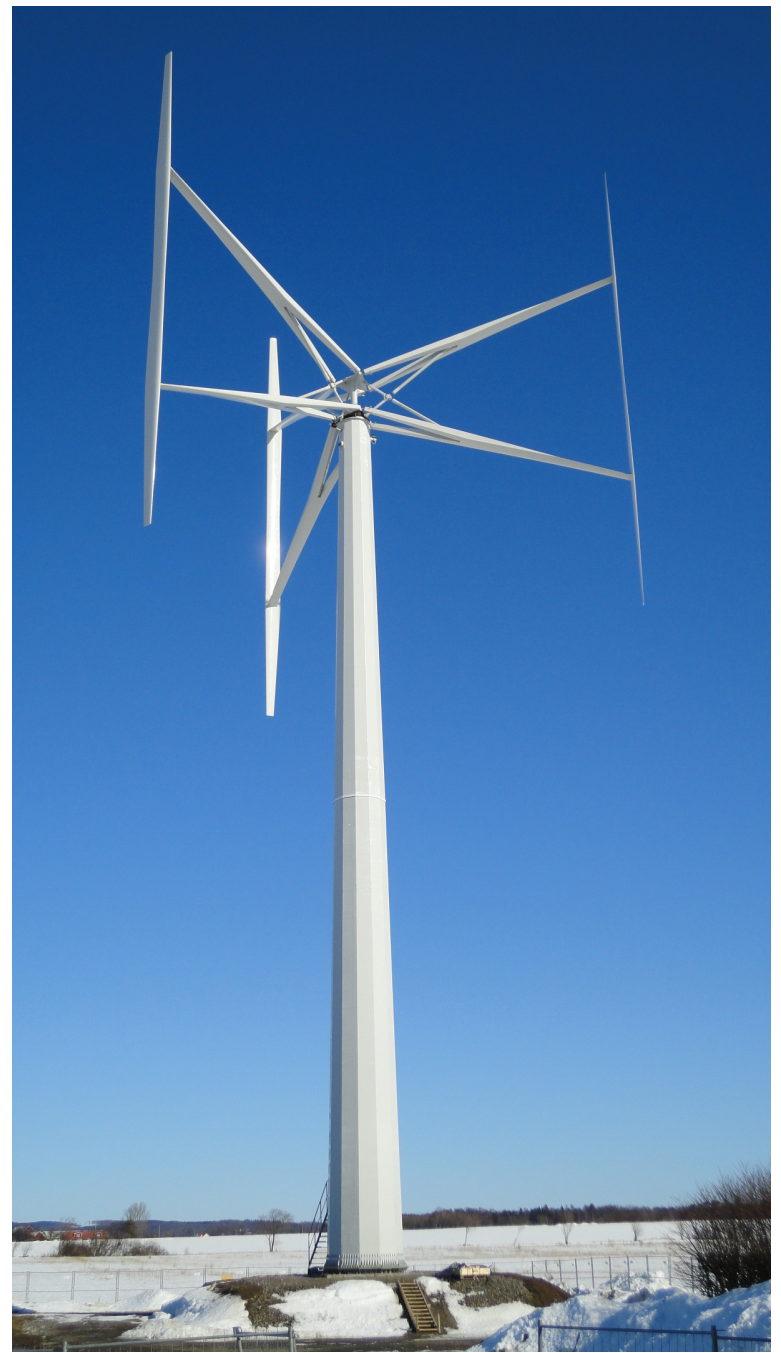

Figure 1. The $200 \mathrm{~kW}$ reference turbine, located in Falkenberg, Sweden.

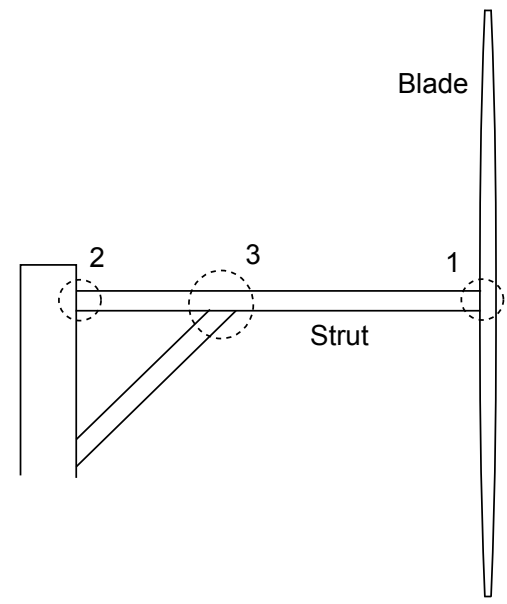

Figure 2. Schematic picture of a blade and supporting strut with simplified geometry. Scalability at the numbered joints are discussed in the text. The additional supporting strut connects at an angle of $45^{\circ}$. 


\subsection{Forces at joint 1}

Considering the nominal turbine in Table I at nominal wind speed, the forces at joint 1 may be estimated as follows. The centripetal force is $F_{c}=m_{b} a \sim 5.6 \times 10^{4} \mathrm{~N}$, where $m_{b}$ is the blade mass, using the nominal tip speed ratio $\lambda$ of about 3.8 . The gravitational force is vertical and amounts to $F_{g}=m_{b} g \sim 3.4 \times 10^{3} \mathrm{~N}$. The aerodynamic force can be approximated by $F_{a}=\frac{1}{2} C_{\text {tot }} \rho S_{b} v^{2}$, where $C_{\text {tot }}=\sqrt{C_{L}^{2}+C_{D}^{2}}, C_{L}$ and $C_{D}$ being the lift and drag coefficients, $\rho$ is the density of air, $S_{b}$ is the blade area and $v$ is the relative wind speed at the blade. Maximum $F_{a}$ is estimated to occur when $C_{\text {tot }} \sim 1$ and $v \sim(\lambda+0.5) U$, and we get $F_{a, \max } \sim 2.9 \times 10^{4} \mathrm{~N}$. This force will add to the centripetal force in the horizontal plane. The material stress due to the radial component of the resulting force is in the range of $\sigma \sim\left(F_{c} \pm F_{a, \max }\right) / A$. (This is a rough estimate; in reality the aerodynamic force on the downwind side is smaller than on the upwind side; it may also be unsymmetrical along the blade, and with two struts the stress at each joint may temporarily be larger.)

As the stress varies cyclically, the joint needs to be designed with respect to fatigue. For a zero mean alternating stress $\sigma_{e}$ the ultimate stress should be at least $\sigma_{u}=k \sigma_{e}$, where $k \sim 10$ to allow for a desired lifetime of $10^{8}-10^{9}$ cycles, which is the typical number of cycles rotor components in a wind turbine have to withstand. The magnitude of $k$ is estimated from measurements on fiberglass composites [10], which is the typical fatigue-prone material at the joints in this design. For a carbon fiber design, $k \sim 10$ is assumed to still apply. If the mean stress $\sigma_{m} \neq 0$ we assume for simplicity that the material is symmetric (which is only approximately true for fiberglass composites) and that Goodman's rule (with unit exponent) can be used,

$$
\sigma_{a}=\sigma_{e}\left(1-\left|\sigma_{m}\right| / \sigma_{u}\right)
$$

where $\sigma_{a}$ is the allowed stress amplitude. Thus, having non-zero mean stress means that the ultimate stress has to be $\sigma_{u}=k \sigma_{a}+\left|\sigma_{m}\right|$. In this particular case we need to design for an ultimate radial stress $\sigma_{u, x}=\left(k F_{a, \max }+F_{c}\right) / A \sim$ $3.4 \times 10^{5} / A \mathrm{~Pa}$. Assuming that a similarly sized area $A$ is relevant for the vertical stress as well, the corresponding ultimate stress to design for is $\sigma_{u, z}=F_{g} / A \sim 3.4 \times 10^{3} / A \mathrm{~Pa}$, as the gravitational force is static. (It might appear strange to compare vertical and horizontal stresses here; however, keeping in mind that for a realistic strut design the struts connect to the blade at an angle, the comparison is more reasonable.) The gravitational contribution to the design ultimate stress becomes of equal size to the aerodynamic contribution at $\alpha \sim 80$, which represents a turning point at which the gravitational force is starting to become the main design driver with respect to dimensioning this joint.

In the intermediate range (from nominal wind speed up to shut down) where the turbine is assumed to be stall controlled at constant power, the rotational speed is more or less constant and hence the centripetal force is unchanged. The aerodynamic force will be somewhat larger than at nominal speed, and, in particular, vary more due to dynamic stall. However, operation below nominal speed is much more likely than above, so looking at nominal wind speed for the entire lifetime will give a fairly good indication, which suggests that scaling up to $\alpha \sim 80$ will be possible. This is, of course, a highly unrealistic scale; in particular, the proposed scaling scheme is likely to break down well below this scale. As presented below, the turning points for the other joints will occur at more realistic scales.

The storm case should be considered as well. With the turbine parked, $F_{c}=0$ and $F_{a} \sim 7 \times 10^{4} \mathrm{~N}$ for maximum $C_{\text {tot }} \sim 1.8$ and $v=U=60 \mathrm{~m} / \mathrm{s}$. It is clear that this implies stresses well below the ultimate stress that was needed to handle fatigue, as discussed above. It is difficult to asses the fatigue implication of storm loads, which may arise from turbulence in the wind and flutter. However, considering the factor of 5 difference between the maximum stress and the ultimate stress, and that the storm loads are present only for a small fraction of the lifetime, the design ultimate stress is expected to be sufficient.

\subsection{Forces at joint 2}

Considering the impact of the gravitational force as compared to the other forces, the situation is more severe at joint 2 and, in particular, at joint 3 . At joint 2 , let $\boldsymbol{F}_{h}$ denote the force from the hub on the strut. We assume the simplified geometry as indicated in Figure 2, with strut mass $2 m_{b}$ and strut center of mass at $r=R / 3$, where joint 3 is also assumed to be 
located. The components of $\boldsymbol{F}_{h}$ become

$$
F_{h z}=-2 m_{b} g, \quad F_{h x}=-\frac{5}{3} m_{b} \Omega^{2} R-F_{a}-5 m_{b} g
$$

where the $z$-direction is vertical and the $x$-direction is along the strut. The last term of $F_{h x}$ arises in order to balance the vertical component of the force from the supporting strut at joint 3. By the same reasoning as given above in section 3.1, joint 2 has to be designed for an ultimate stress of $\sigma_{u, x}=\left(k F_{a, \max }+5 / 3 m_{b} \Omega^{2} R+5 m_{b} g\right) / A$. All three contributing terms scale differently, and at $\alpha \sim 17$ the gravitational term takes over as the most important contribution. (An average lever arm for $F_{a}$, as discussed below in section 3.3, could be introduced here as well; this would increase $\alpha$ somewhat.)

At storm conditions with a parked turbine, $\Omega$ is zero. The turbine has to be designed for extreme gusts with considerable wind shear. Therefore, an unsymmetrical loading is assumed for the storm case, where the aerodynamic force $F_{a}$ has a lever $\operatorname{arm} b / 4$ to the midspan, where $b$ is the blade span. Assuming unit turbine aspect ratio, which means that $b=2 R$, we get

$$
F_{h z}=-\frac{3}{2} F_{a}-2 m_{b} g, \quad F_{h x}=-\frac{5}{2} F_{a}-5 m_{b} g .
$$

With maximum aerodynamic load at storm being $7 \times 10^{4} \mathrm{~N}$ as above, we note that the storm stress is well below the ultimate stress.

\subsection{Forces at joint 3}

Turning finally to joint 3 , we first consider a symmetric aerodynamic load $F_{a}$. Denoting the force from the supporting strut on the main strut by $\boldsymbol{F}_{s}$, its components are

$$
F_{s z}=F_{s x}=5 m_{b} g
$$

i.e. they are determined only by the mass of the blade-strut system. However, the aerodynamic load will always have an asymmetric component due to turbulence and shear in the wind field. Introducing just a little lever arm on $F_{a}$ will result in a significant effect on this joint. It is therefore necessary to design this joint with respect to an aerodynamic load where the lever arm is non-zero. Denoting the lever arm by $a$, the vertical component becomes

$$
F_{s z}=5 m_{b} g+\frac{6 a}{b} F_{a}
$$

The lever arm may be estimated from measurements on fluctuations of vertical wind shear [11], where we additionally assume that the lateral wind speed (affecting the wind direction) fluctuates on the same level as the longitudinal wind speed. A change in wind direction affects the angle of attack, which is proportional to aerodynamic force. The wind direction gradient can in this way be related to a lever arm on the total force. Assuming a standard deviation of the vertical speed gradient of $0.02 \mathrm{~s}^{-1}$, which is in the lower regime, we get an average lever arm of $0.08 b$. This indicates that $\sigma_{u}=\left(0.08 \times 6 k F_{a, \max }+5 m_{b} g\right) / A$, and according to the previous estimate of the aerodynamic force the terms in $\sigma_{u}$ becomes of equal influence at $\alpha \sim 7.8$.

At storm conditions, assuming the load acts at the 1/4 chord location, and utilizing the previously estimated storm aerodynamic load, we obtain a stress close to the ultimate stress needed for nominal operation determined in the paragraph above. Hence, it is likely that at this joint the storm load will determine the design strength. It is reasonable that fatigue considerations will put some factor in front of the aerodynamic load so that the $\alpha$ will still be in the same regime or above. However, due to uncertainties in judging the fatigue implications here, a conservative statement is that this type of scaling is reasonable up to $\alpha \sim 7$.8. In terms of power rating this implies an upper scale rating of about $30 \mathrm{MW}$. The data for this hypothetical turbine is given in Table II.

Compared to the reference design, the calculation is based on a $30 \%$ weight reduction of the blades and struts, as explained in the end of section 2. Performing the calculation without this weight reduction, i.e. assuming standard fiberglass blades, the structural upper scale becomes $\alpha \sim 5.3$, corresponding to a rating of $11 \mathrm{MW}$. 
Table II. Data for the upper-size turbine

\begin{tabular}{ll}
\hline \hline Power rating & $30 \mathrm{MW}$ \\
Radius $(R)$ & $100 \mathrm{~m}$ \\
Hub height & $310 \mathrm{~m}$ \\
Blade mass (one blade) & $300 \times 10^{3} \mathrm{~kg}$ \\
Nominal wind speed $(U)$ & $16 \mathrm{~m} / \mathrm{s}$ \\
Nominal RPM & 5.7 \\
\hline
\end{tabular}

The number of cycles that a turbine experiences during its lifetime is typically proportional to rotational speed $\Omega$; as a consequence, a bigger turbine may be designed to withstand a slightly smaller number of cycles. However, the effect is small and neglected in this estimate. Also, fatigue issues need a much more thorough treatment. The present calculation is just intended as a rough indication of the scale at which gravity becomes important.

Special treatment of joint 3 is needed at and above $\alpha \sim 7.8$. Likely, design improvements at joint 3 will render the design viable at somewhat larger scales. However, well above this scale, we expect major difficulties in the design work, as an oversized joint may be accompanied by an aerodynamic degradation.

\subsection{Shear stress in the shaft}

The reference design includes a shaft that transfers the torque $M_{z}$ from the turbine to a ground level generator. The main shaft is modelled as a thin-walled circular beam, and the shear stress is given by

$$
\tau=\frac{M_{z}}{2 \pi r^{2} t}
$$

where $r$ is the radius and $t$ is the thickness of the beam. According to the proposed scaling scheme, $t$ is to scale as $\alpha^{1+2 n}$. Since $M_{z}=P / \Omega$ we conclude that the shear stress in the shaft is scale invariant. The shear stress has to be lower than the buckling limit, which is in the range of [12]

$$
\tau^{\prime}=\frac{E}{1-\nu^{2}}\left(\frac{t}{H}\right)^{2}\left(-2.39+\sqrt{96.9+0.605 \beta^{1.5}}\right)
$$

where $E$ is the Young's modulus, $\nu$ is the Poisson's ratio for the material, and $\beta=\sqrt{1-\nu^{2}} H^{2} / t r$. As $t$ scales as $\alpha^{1+2 n}$, and $H$ and $r$ as $\alpha$, it is clear that the shear limit for buckling will increase with $\alpha$, and the proposed scaling scheme is viable with respect to the main shaft.

\subsection{Eigenfrequencies}

It is interesting to examine the scaling of eigenfrequencies of the scaled turbine as compared to the rotational frequency. Consider the oscillations due to bending of the hub shaft (the uppermost part of the shaft). The hub shaft is modelled as a thin-walled circular beam with second moment of inertia $I_{y}=\pi r^{3} t$, where $r$ and $t$ now denotes the radius and thickness of the hub shaft. Again, $t$ is to scale as $\alpha^{1+2 n}$. The moment $M_{x}$ from the hub shaft on the turbine due to the deviation $\theta$ of the turbine position from the horizontal plane is given by

$$
M_{x}=E I_{y} \theta / h
$$

where $h$ is the effective height of the hub shaft. For a parked turbine, assuming a rigid turbine with moment of inertia $I_{t x}$ along a horizontal axis, the equation of motion for this type of oscillation is $I_{t x} \ddot{\theta}=-M_{x}$ and the frequency becomes

$$
\omega_{1}=\sqrt{\frac{E I_{y}}{I_{t x} h}} .
$$


Since $I_{y}$ scales as $\alpha^{4+2 n}, I_{t x} \sim m R^{2}$ as $\alpha^{5+2 n}$, and $h$ as $\alpha$, we note that $\tilde{\omega}_{1}=\alpha^{-1} \omega_{1}$, which is not exactly the same scaling as $\Omega$. For a rotating turbine the frequency $\omega_{1}$ will shift as a function of $\Omega$ (see [13]), which will change the scaling slightly, but there will still be a difference in scaling. The result is similar for the main tower frequency and other oscillations in the turbine. Hence, resonance analysis against the per rev frequencies is not scale invariant and need to be readdressed for different scales. It is generally the case that the eigenfrequencies in this scaling scheme decrease faster than $\Omega$ does. This implies that special care need to taken, during up-scaling, regarding eigenfrequencies with resonances just above the operational rpm range.

\section{CONCLUSIONS}

The gravitational force has a worse scaling behaviour than the aerodynamic force and this is expected to accelerate the cost of machines above some upper scale. The estimates performed in this note suggest that the structural upper scale for the $\mathrm{H}$-rotor is in the range of $30 \mathrm{MW}$. This is the scale where gravity starts to become of major influence when dimensioning key joints. The eigenfrequencies will shift with respect to rotational frequency, for the present scaling scheme, so that possible resonances have to be readdressed for different scales.

The upper scale proposed here should not be viewed as the most economic scale, a scale which is much more complicated to calculate and involves market, volume and manufacturing considerations. The scale proposed here is likely to put an upper bound on the most economic scale, as the rate at which the cost increases is likely to accelerate above this upper scale.

\section{ACKNOWLEDGEMENTS}

This work was conducted within the STandUP for ENERGY strategic research framework. The Swedish Energy Agency and VINNOVA are acknowledged for supporting the Swedish Centre for Renewable Electric Energy Conversion.

\section{REFERENCES}

1. Caduff M, Huijbregts MAJ, Althaus H, Koehler A, Hellweg S. Wind power electricity: The bigger the turbine, the greener the electricity? Environmental Science and Technology 2012; 46(9):4725-4733.

2. Sieros G, Chaviaropoulos P, Sørensen JD, Bulder BH, Jamieson P. Upscaling wind turbines: theoretical and practical aspects and their impact on the cost of energy. Wind Energy 2012; 15(1):3-17.

3. Lackner MA, van Kuik G. A comparison of smart rotor control approaches using trailing edge flaps and individual pitch control. Wind Energy 2010; 13(2-3):117-134.

4. Eriksson S, Bernhoff H, Leijon M. Evaluation of different turbine concepts for wind power. Renewable and Sustainable Energy Reviews 2008; 12(5):1419 - 1434.

5. Sutherland H, Berg D, Ashwill T. A retrospective of VAWT technology. Report No. SAND2012-0304, Albuquerque, New Mexico: Sandia National Laboratories 2012; .

6. Musgrove P. Wind energy conversion: Recent progress and future prospects. Solar \& Wind Technology 1987; 4(1):37 -49 .

7. Kjellin J, Eriksson S, Bernhoff H. Electric control substituting pitch control for large wind turbines 2012; Submitted to Renewable Energy.

8. Katz J, Plotkin A. Low-Speed Aerodynamics. Cambridge Aerospace Series, Cambridge University Press, 2001. 
9. Griffin DA. Cost \& performance tradeoffs for carbon fibers in wind turbine blades. SAMPE Journal-Society for the Advancement of Material and Process Engineering 2004; 40(4):20-28.

10. Sutherland H. On the fatigue analysis of wind turbines. Report No. SAND99-0089, Albuquerque, New Mexico: Sandia National Laboratories 1999; .

11. Bergström H. A statistical analysis of gust characteristics. Boundary-Layer Meteorology 1987; 39:153-173.

12. Young WC, Budynas RG. Roark's formulas for stress and strain, vol. 6. McGraw-Hill New York, 2002.

13. Ottermo F, Bernhoff H. Resonances and aerodynamic damping of a vertical axis wind turbine. Wind Engineering 2012; 36:297-304. 